\title{
Norois
}

Environnement, aménagement, société

Dynamiques fluviale et littorale, activité viticole

\section{Intérêts et limites des données « CatNat » pour un inventaire des inondations. L'exemple des « crues rapides » liées à de violents orages (Bassin parisien, Nord de la France)}

Interests and limits of the French "CatNat" data for inventorying floods. The example of the "flash floods" linked to violent thunderstorms (Paris Basin, North-western France)

Johnny Douvinet

\section{OpenEdition}

Journals

Édition électronique

URL : https://journals.openedition.org/norois/1733

DOI : $10.4000 /$ norois. 1733

ISBN : 978-2-7535-1549-9

ISSN : $1760-8546$

Éditeur

Presses universitaires de Rennes

Édition imprimée

Date de publication : 1 décembre 2006

Pagination : $17-30$

ISBN : 978-2-7535-0407-3

ISSN : 0029-182X

Référence électronique

Johnny Douvinet, «Intérêts et limites des données « CatNat » pour un inventaire des inondations. L'exemple des « crues rapides » liées à de violents orages (Bassin parisien, Nord de la France) », Norois [En ligne], 201 | 2006/4, mis en ligne le 01 décembre 2008, consulté le 13 janvier 2022. URL : http:// journals.openedition.org/norois/1733; DOI : https://doi.org/10.4000/norois.1733 


\title{
INTÉRÊTS ET LIMITES DES DONNÉES « CATNAT » POUR UN INVENTAIRE \\ DES INONDATIONS. L'EXEMPLE DES « CRUES RAPIDES » \\ LiÉES À DE VIOLENTS ORAGES (BASSIN PARISIEN, NORD DE LA FrANCE)
}

\author{
JohnNy DOUvinet \\ GÉOPHEN - LETG UMR 6554 CNRS \\ (Université de Caen Basse-Normandie), \\ Esplanade de la Paix - 14032 CAEn cedex 5 \\ johnny.douvinet@unicaen.fr
}

\section{RÉSUMÉ}

Depuis deux décennies, des phénomènes de "crues rapides » liés à de violents orages sont fréquemment observés sur les plateaux limoneux du Bassin parisien (Nord de la France). Ces crues souvent appelées à tort « coulées de boue » ou «crues turbides » sont toujours associées à du ruissellement érosif. Quoique fréquentes, elles n'en restent pas moins mal connues et peu étudiées sur ces régions. L'objectif de cet article est de proposer un inventaire des inondations causées par ces "crues rapides » à partir de la base "CatNat» dans le but de mieux connaître leur occurrence spatiale. D'un point de vue scientifique, l'exploitation de la base de données permet de travailler uniquement sur la sinistralité des zones touchées. La démarche consiste à traiter cette base statistique en vue de créer notre propre base de données, puis une fois spatialisées, ces données permettent de localiser les communes où le nombre de déclarations est le plus élevé. Des problèmes imputables aux objectifs réglementaires et à l'architecture de la base de données apparaissent toutefois rapidement et en limitent son utilisation. Il devient alors indispensable d'analyser les dossiers présentés devant la commission interministérielle qui statue sur la reconnaissance de l'état de "catastrophe naturelle». Suivant les informations récoltées, nous pouvons sélectionner uniquement les «crues rapides » et dresser un inventaire détaillé des zones sinistrées tout en affinant considérablement l'analyse statistique initiale.

MotS CLÉS : Bassin parisien - Crues rapides - Inondations - Inventaire - Sinistralité.

\section{ABSTRACT}

Interests and limits of the French "CatNat" data for inventorying floods. The example of the "flash floods" linked to violent thunderstorms (Paris Basin, North-western France)

Over the last two decade "flash floods" due to violent thunderstorms have currently happened on the loamy plateaux of the Paris Basin (North-western France). Traditionally designed as "muddy floods", these floods have often been related to paroxystic soil erosion. Nevertheless, even if such hydrological events are frequently observed on these cultivated areas they remain poorly known. The aim of this study is to improve our knowledge about the spatial extend of these "flash floods". Our research is based on the analysis of the French 
"CatNat" data base which allows only working on flooded places. A statistical analysis permits localizing areas where local bodies are strongly demanding to the central power that a state of "catastrophic disaster" is declared. However, the structure of the data base raises some difficulties, more especially regarding legal issues. More information is obtained when the arguments for the "CatNat" decision are analysed. Such approach allows filling many gaps of the "CatNat" data base, building an inventory of "flash floods" and to better the results of the former analysis.

KEY WORDS : Flash Floods - Flooded Places and Damage - Inventory - Paris Basin.

Des « crues rapides » liées à de violents orages printaniers et estivaux apparaissent de manière récurrente sur les plateaux limoneux du Bassin parisien (Nord de la France). Leur ampleur a fortement augmenté depuis le début des années 1990, en particulier dans la région du Pays de Caux à l'Ouest de la Seine-Maritime (MATE, 2001 ; Delahaye, 2002 ; Le Bissonnais et al., 2002). Ces crues sont souvent associées à du ruissellement érosif (Ludwig, 1992; Auzet et al., 1995; Angeliaume et Wicherek, 1997; Meyer, 2001 ; Cerdan et al., 2002; Boardman et al., 2003). Cette association est plus qu'évidente puisque la majeure partie du matériel charrié alimentant la crue provient de l'érosion des sols. Des travaux récents (Hauchard et al., 1999; MATE, 2001 ; Delahaye, 2002; Douvinet et al., 2006a) démontrent toutefois à quel point la morphologie est le principal facteur de contrôle de la dynamique de ces « crues rapides » à fort ruissellement de surface, en relation, bien sûr, avec des pluies importantes $(>50 \mathrm{~mm})$ tombant sur un laps de temps assez court (de l'ordre de quelques heures seulement). La localisation des surfaces ruisselantes, les modifications du parcellaire et les pratiques agraires n'aggraveraient qu'en second lieu les effets dynamiques induits par la topographie (Douvinet et al., 2006b).

Même si les conséquences économiques et sociales sont de plus en plus importantes et visibles, il n'est pas certain que ces aléas augmentent en nombre et en fréquence sur le moyen terme. L'occurrence spatiale de ces événements reste également encore mal connue et surtout peu étudiée. L'objectif ici est donc de dresser un inventaire des inondations causées par ces phénomènes sur l'ensemble du Bassin parisien. Les sources pour réaliser ce type de travail sont aussi nombreuses que diversifiées (coupures de presse, archives, base «CatNat», déclarations aux assurances, etc.). Par souci d'homogénéité entre les informations, nous nous sommes tournés vers la base de données «CatNat », base élaborée par le ministère de l'Écologie et du Développement Durable, où sont recensées depuis le $1^{\text {er }}$ janvier 1983 les reconnaissances de l'état de « catastrophe naturelle » pour toutes les communes françaises. L'inventaire sera ainsi basé sur une évaluation des sinistres et sur la notion d'endommagement (Pigeon, 2002) mais ne peut prétendre à la définition de «zones à risque » puisque l'aléa restera négligé.

La première étape (1) consiste à traiter la base "CatNat » afin de créer notre propre base de données sur les inondations liées à des épisodes orageux. Son interprétation statistique et spatiale (2) permet ensuite de localiser les communes pour lesquelles les demandes ont été les plus fortes depuis 1983. Des problèmes (3) inhérents à la base de données et à la prise de décision de l'état de « catastrophe naturelle » surgissent toutefois rapidement et remettent en cause la fiabilité des données. Il est alors nécessaire d'analyser (4) les dossiers élaborés par les communes pour obtenir la reconnaissance de l'état de « catastrophe naturelle ». Suivant la nature des informations retrouvées, nous pouvons désormais mieux évaluer l'importance des crues et en mesurer la gravité, mais surtout proposer un inventaire complet des zones sinistrées tout en affinant de manière importante les résultats donnés par la base de données initiale (5). 


\section{Un inventaire basé sur la sinistralité}

\section{CRÉATION D’UNE BASE DE DONNÉES « INONDATIONS ET COULÉES DE BOUE » LIÉES À DES ORAGES SUR LES RÉGIONS DE PLATEAUX DU NORD DE LA FRANCE}

Les événements indemnisés au titre de la reconnaissance de l'état de « catastrophe naturelle »

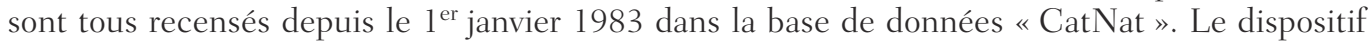
juridique de cette reconnaissance est amplement détaillé dans la circulaire du 19 mai 1998 et a été récemment complété par la loi Bachelot du 13 août 2004. L'intérêt de travailler à partir de ces données est de considérer l'inondation comme un phénomène dommageable. Pour le phénomène inondation, la prise de décision est positive lorsque l'intensité du phénomène est reconnue comme « anormale », c'est-à-dire lorsque l'intensité journalière des pluies observées est supérieure à la valeur décennale calculée sur les données des stations Météo France proches de la zone concernée par l'épisode pluvieux. Les relevés des stations bénévoles et de quelques particuliers peuvent compléter les données des stations officielles qui, la plupart du temps, ne se situent pas sous l'épicentre de l'orage. L'intégration des lames d'eau grâce aux échos-radars des stations du réseau ARAMIS de Météo France permet depuis plusieurs années de visualiser le passage des zones pluvieuses les plus intenses mais aussi de vérifier la fiabilité des relevés à différents pas de temps (Météo France et MEDD, 2004). L'imagerie radar était déjà disponible dès 1986. La taille des pixels des images est récemment passée de $10 \mathrm{~km}^{2}$ à l'échelle du km² en 2000.

Parmi les items des événements couverts par l'assurance « dommages ${ }^{1}$ », seules les « inondations et coulées de boue » ont été gardées (fig. 1). Les données des huit départements faisant partie de la zone d'étude ${ }^{2}$ ont ensuite été sélectionnées. Puis seuls les épisodes apparus entre les mois de mai et août ont été choisis. Cette période est considérée comme étant la plus propice à l'apparition des orages les plus violents. Les épisodes apparus durant les autres mois ont été volontairement exclus de l'analyse, à l'exception de quelques épisodes ayant eu lieu durant les premiers jours de septembre ( ${ }^{\text {er }}$ septembre 1987, 2 septembre 1992; 3 septembre 1998; 6 septembre 1999). Les redondances avec des items proches et les événements ayant fait l'objet de plusieurs arrêtés ont aussi été supprimées. Au final, il reste 3568 déclarations (fig. 1).

\section{INTERPRÉTATION DE LA BASE « CATNAT » ET SPATIALISATION DES DONNÉES}

L'analyse de la base de données permet de mesurer la sensibilité des départements. Un rapport de 1 à 10 est utilisé pour pouvoir comparer le nombre des épisodes aux déclarations. La SeineMaritime apparaît la plus touchée (fig. 2) : on dénombre 720 déclarations et 42 épisodes sur la période 1983-2005, soit en moyenne 17 déclarations par épisodes. L’Aisne complète ce premier groupe avec 614 inondations. Les épisodes sont plus nombreux (64) mais touchent moins de communes (10 en moyenne). On y recense même $43 \%$ de toutes les reconnaissances déclarées entre 1983 et 1988, ce qui montre combien ce phénomène était déjà présent durant cette période. Un deuxième groupe est composé du Nord, de l'Oise et du Pas-de-Calais. Les épisodes sont proches, entre 52 et 57 , tout comme le nombre de déclarations qui oscille aux alentours de 450 . Les autres départements (Calvados, Eure, Somme) sont bien moins touchés.

1. Toutes les personnes qui possèdent pour leurs biens une assurance «pertes d'exploitation » ou « dommages » sont automatiquement couvertes en cas de dommages causés à ces biens dus aux « forces de la nature » : inondation, glissement de terrain, sécheresse, avalanche, tremblement de terre. Sont exclus depuis le 25 juin 1990 les dommages résultant des effets du vent dû aux tempêtes, ouragan et cyclones. La sécheresse devrait aussi prochainement disparaître de la procédure d'indemnisation, notamment à la suite des « atermoiements de l'État face à l'indemnisation de la sécheresse de 2003 » qui met à jour certaines limites du système français, en particulier l'absence de réserves financières suffisantes pour couvrir une catastrophe majeure (MEDD, 2005).

2. La zone d'étude regroupe les trois régions du Nord de la France reconnues comme étant particulièrement sensibles aux «coulées de boue» (Le Bissonnais et al., 2002). Nous avons choisi d’y ajouter le département du Calvados car ce genre d'épisode, qui restait a priori inconnu dans cette région, y a été récemment observé ( ler $^{\text {juin }} 2003$ à Trouville). 
1 Base de données initiale: les items de la base "CatNat"

"affaissement de falaise"
"avalanche"
"chocs mécaniques liées à l'action des vagues"
"chutes de rochers/blocs rocheux"
"coulées de boue" (14/02/1990)
"coulées de boue et laves torrentielles"
"crues torrentielles et glissement de terrain"
"éboulement, glissement et affaissement de terrain"
"éboulements rocheux"
"effondrement de terrain"
"effondrements/éboulements"
"glissement de terrain"
"inondations et chocs mécaniques liées à l'action des vagues"
"inondations et coulées de boue"
"inondations par remontée de nappe phréatique"
"inondations, chocs mécaniques liés à l'action des vagues et glissement de terrain"
"inondations, coulées de boue et effets exceptionnels dus aux précipitations" (22/01/1992)
"inondations, coulées de boue et mouvement de terrain" (25/12/1999)
"mouvements de terrain"
"mouvements de terrain consécutifs à la sécheresse"
"poids de la neige/chutes de neige"
"séisme"
"tassement de terrain"
"tempernade et grêle"
"vents cycloniques"

2 "inondations et coulées de boue"

(1983-2004)

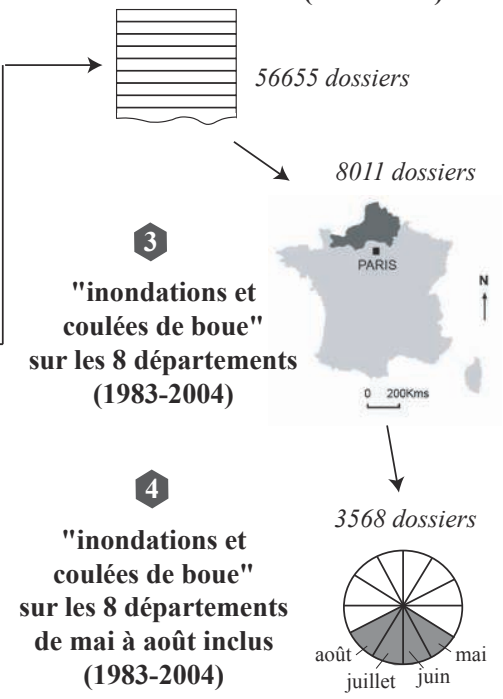

Figure 1 : Création de notre base de données à partir de la base nationale "CatNat »

Creation of our data base according to the national French "Catnat" data base

Il convient toutefois de pondérer ces résultats par rapport au nombre de communes et à la surface de chaque département afin de ne pas perturber l'interprétation spatiale (Delahaye, 1999). Les orages affectent une surface moyenne deux fois plus grande (tableau 1) en SeineMaritime $\left(154 \mathrm{~km}^{2}\right)$ que dans le Nord ou dans l'Aisne, voire trois fois plus que dans le Pas-deCalais $\left(53 \mathrm{~km}^{2}\right)$. Les données ont dans un second temps été spatialisées afin de mieux connaître la répartition spatiale des communes touchées. La carte (fig. 3) met ainsi en évidence l'emprise géographique de ces événements et permet d'identifier des zones d'impact assimilables à des «épicentres » (Le Bissonnais et al., 2002). Le Pays de Caux ressort comme la région de loin la plus fortement touchée. Les communes à plus de 4 reconnaissances y sont toutes localisées. D'autres régions agricoles apparaissent secondairement : les collines de l'Artois (au nord-ouest d'Arras); le Plateau Picard (au sud-est d'Amiens); le Vexin (au sud-ouest de l'Eure); le Tardenois (au sud de l'Aisne); le Saint-Quentinois (au nord de l'Aisne); le Soissonnais et le Valois (au sud de l'Aisne et à l'est de l'Oise); le Plateau de Neubourg (centre de l'Eure).

Des problèmes apparaissent toutefois rapidement à la lecture de la carte (fig. 3). Les « crues rapides » regroupées dans l'item « inondations et coulées de boue » peuvent facilement se confondre avec des phénomènes hydrologiques très différents. Le ruissellement urbain ou la stagnation des eaux pluviales font par exemple ressortir les pôles miniers et urbains du Nord de la France. Les villes les plus importantes sont également celles où le nombre d'arrêtés est le plus élevé, tout simplement à cause d'une plus forte vulnérabilité. Les communes semblent par ailleurs être situées sur des axes préférentiellement définis par les épisodes orageux. 


\begin{tabular}{|c|c|c|c|c|c|c|c|c|c|}
\hline & 14 & 76 & 27 & 80 & 60 & 62 & 59 & 02 & moyenne \\
\hline $\begin{array}{l}\text { Déclarations entre } \\
\text { mai et août inclus (A) }\end{array}$ & 311 & 720 & 294 & 309 & 424 & 424 & 472 & 614 & 446 \\
\hline $\begin{array}{l}\text { Nombre d'épisodes } \\
\text { recensés }(\mathrm{B})\end{array}$ & 26 & 42 & 36 & 36 & 57 & 55 & 52 & 61 & 45,6 \\
\hline $\begin{array}{l}\text { Communes déclarées } \\
\text { par épisode (A/B) }\end{array}$ & 11,9 & 17,1 & 8,2 & 8,6 & 7,4 & 7,7 & 9,1 & 10,0 & 10,0 \\
\hline $\begin{array}{l}\text { Nombre de } \\
\text { communes par } \\
\text { département }\end{array}$ & 704 & 745 & 675 & 783 & 693 & 894 & 653 & 816 & / \\
\hline $\begin{array}{l}\text { Surface du } \\
\text { département }\left(\mathrm{km}^{2}\right)\end{array}$ & 6334 & 6721 & 7436 & 5562 & 6211 & 5901 & 5773 & 6044 & / \\
\hline $\begin{array}{l}\text { Surface moyenne } \\
\text { touchée par épisode } \\
\text { par département } \\
\left(\mathrm{km}^{2}\right)\end{array}$ & 107 & 154,3 & 90,3 & 61,1 & 66,3 & 52,6 & 80,4 & 74,1 & 85,8 \\
\hline
\end{tabular}

Tableau 1 : Nombre d'épisodes et de déclarations entre mai et août pour chaque département (numéroté sur la figure 3) reconnues entre 1983 et 2005 d'après la base « CatNat »

Number of floods and of recognized events inventoried between May and August on each department (numbered on figure 3) over the period 1983-2005 from to the "CatNat" base

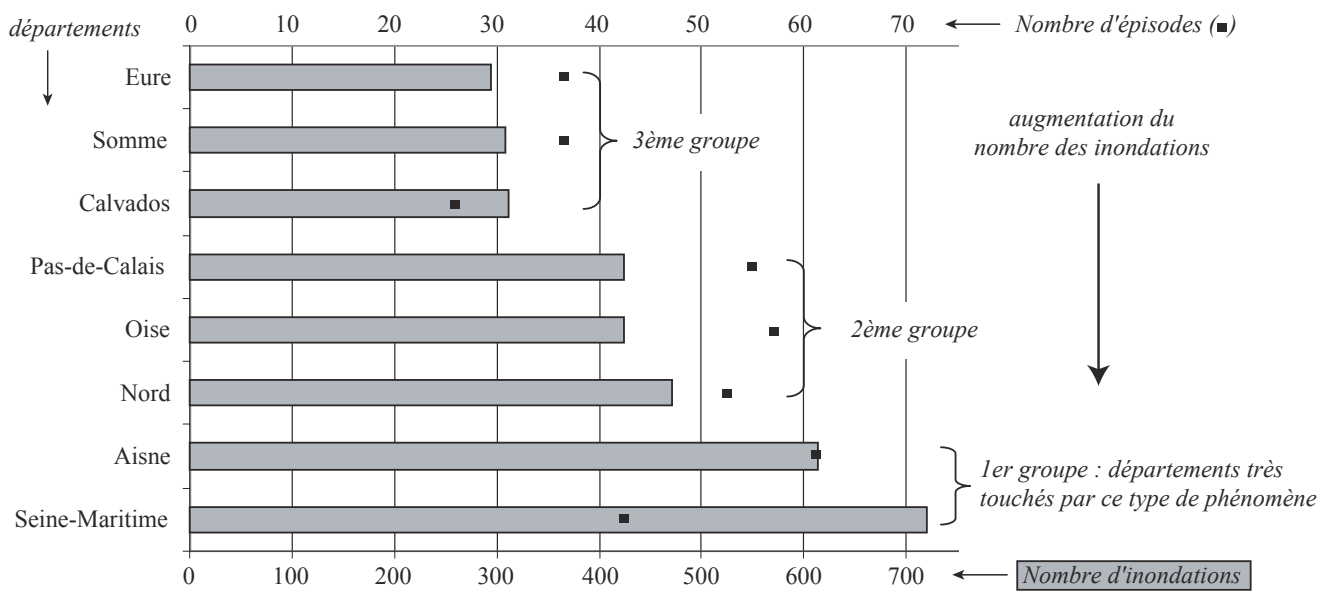

Figure 2 : Nombre de déclarations par département pour l’item « inondations et coulées de boue » recensées entre mai et août sur la période 1983-2005 (d'après la base «CatNat »)

Number of "Floods and muddy floods" recognized by the state of "catastrophic disaster" between May and August over the period 1983-2005 (from the "CatNat" base)

\section{Critiques des sources et limites pour leur exploitation statistique}

L'indemnisation au titre des « catastrophes naturelles » est déjà dès sa mise en place reliée aux politiques de prévention des risques (MEDD, 2005). La base de données sert au suivi des déclarations et permet surtout de cibler les communes où les arrêtés deviennent récurrents. Des limites imputables aux objectifs réglementaires et à l'architecture de la base de données apparaissent pourtant rapidement et limitent l'exploitation scientifique initialement attendue. 


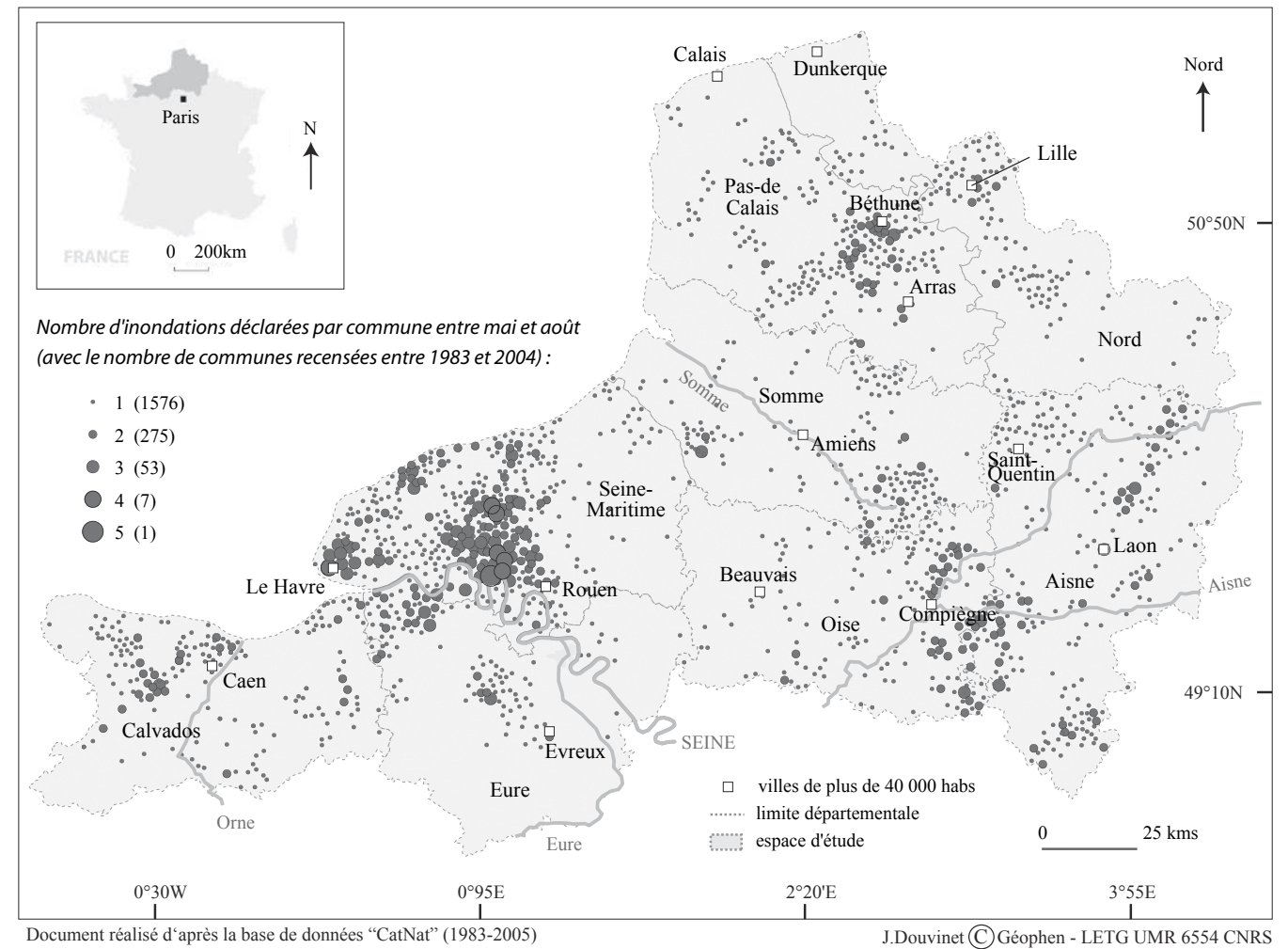

Figure 3 : «Inondations et coulées de boue » reconnues entre mai et août inclus (1983-2005) pour chaque commune à l'échelle de la zone d'étude (d'après la base "CatNat »)

"Floods and muddy floods" occurred between May and August on each commune being part of the studied area over the period 1983-2005 (starting from the "CatNat" base)

\section{UN SEUIL PLUVIOMÉTRIQUE PEU OBJECTIF POUR DES PLUIES ORAGEUSES}

La définition du seuil d'intervention de la garantie à partir de la valeur décennale peut paraître subjective pour des pluies orageuses. Les stations se situent rarement sous l'épicentre orageux car le réseau des stations est assez lâche (Météo-France et MEDD, 2004); les données bénévoles ou celles des particuliers semblent à l'inverse exagérées (fig. 4); l'imagerie radar n'est pas toujours fiable et peut même parfois présenter d'importantes déficiences, comme ce fut le cas lors des fortes pluies orageuses du 4 juillet $2005^{3}$. La valeur décennale n'a également pas beaucoup de signification. La qualification en terme de probabilité/période de retour par la méthode de renouvellement (ou par la loi GEV) nécessite de disposer de référence régionale statistiquement valide, avec des données recueillies sur plus de 30 ans. Or, cette démarche n'est valable que pour $31 \%$ des 586 stations recensées sur la zone d'étude. L'instruction "CatNat" du 15 février 2000 stipule par ailleurs qu' « il n'est pas autorisé de fournir de durée de retour d'une précipitation sur un pas de temps inférieur à une heure et qu'il convient de considérer qu'elle s'est produite en une heure ${ }^{4}$ ». Cependant, ce pas de temps peut faire disparaître des épisodes pour lesquels les dommages liés à des « crues rapides » ont été importants. Les 48 mm tombés en 30 minutes le 23 juin 1984 sur la

3. Des cellules orageuses actives ont été masquées à cause de la présence d'un écran nuageux entre le radar et la zone concernée (Béthune), se conjuguant à l'éloignement de la station radar en limite de portée climatologique.

4. D'après l'article M de la récente loi du 13 août 2004 sur la modernisation de la sécurité civile. 
commune de La Chapelle (Oise) ou les 40 mm tombés en 50 minutes le 22 juillet 2004 à Tupigny (Aisne) n'ont pas fait l'objet d'une reconnaissance car les données, rapportées à l'échelle horaire, repassent en dessous de la valeur décennale ( $\sim 50 \mathrm{~mm})$.

\section{UNE PRISE DE DECISION AVANT TOUT POLITIQUE}

Des doutes importants sont émis quant à la scientificité de la prise de décision. Pour les inondations, la décision repose uniquement sur les notions d'intensité anormale et de cause déterminante du phénomène sans que soient pris en compte le coût des dommages matériels ni même l'ampleur des zones inondées. Le choix est avant tout politique. «Le recours insuffisant à une expertise scientifique incontestable pour définir et caractériser les catastrophes naturelles » (MEDD, 2005) remet toutefois en cause la pertinence de la décision. "Comme ce caractère exceptionnel relève non pas d'un écart à une mesure, mais d'un jugement interministériel, cette définition n'a rien de scientifique : elle est d'ordre politique » souligne A. Dauphiné (2001). Cette situation d'incertitude explique la position de l'État, toujours à la recherche d'une synthèse (on devrait dire un compromis) entre les intérêts contradictoires des assureurs et des assurés, sans oublier ses propres intérêts et ceux des élus.

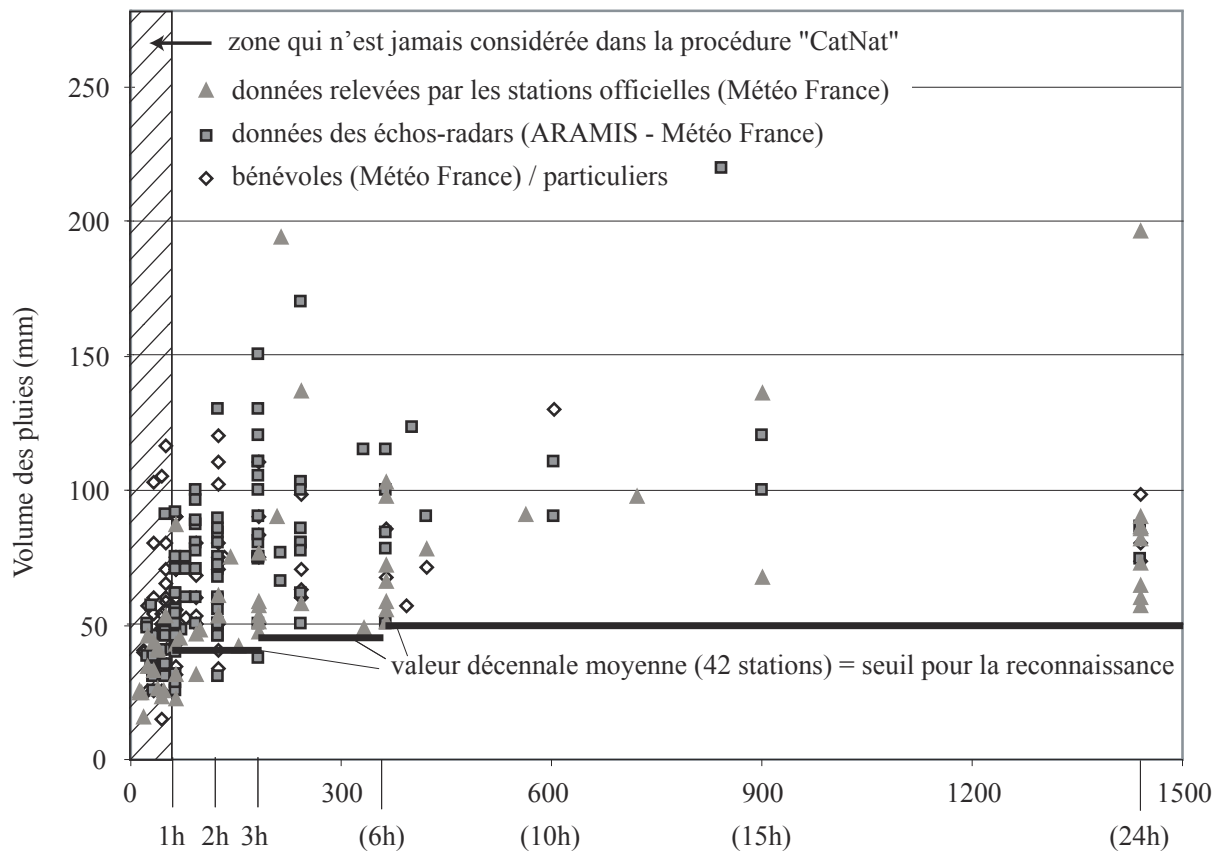

durée des pluies / de l'épisode pluvieux en minutes (ou en heures)

Figure 4 : Nature des données pluviométriques utilisées pour la reconnaissance «CatNat » qui correspondent à des pluies ayant réellement données des " crues rapides " liées à des orages

Nature of the rainfall data used for the state of "natural disaster" for which "flash floods" linked to violent thunderstorms have really happened on the studied area 


\section{DES PROBLÉMES LIÉS À L'ARCHITECTURE DE LA BASE DE DONNÉES}

D'autres problèmes viennent directement de la structure de la base de données. Tout d'abord, l'item «inondations et coulées de boue " regroupe trop de phénomènes hydrologiques distincts : des phénomènes de ruissellement (en milieu urbain et rural), des débordements de cours d'eau, des crues instantanées ainsi que des saturations des réseaux d'évacuation des eaux pluviales. On ne peut donc pas connaître avec précision quels sont les épisodes orageux qui ont uniquement déclenché des «crues rapides ». Par ailleurs, les dégâts liés aux tempêtes et à la grêle sont normalement exclus du régime «CatNat » car ils sont couverts par les assurances privées. Pourtant, la majorité des dommages pour les communes reconnues pour les épisodes du 14 août 1985, du 23 juillet 1988, du 4 juillet 2005 (tempêtes) ou du 24 juillet 1993 et du 6 juin 1998 (grêles) sont dus aux effets combinés du vent et de la grêle. Ces phénomènes étant associés dans l'item « inondations et coulées de boue », il est impossible de ne sélectionner que les communes inondées. Un autre problème vient du fait que l'avis favorable soit rapporté à l'échelon communal. En effet, les inondations concernent souvent une toute petite partie de la commune et non la totalité. Les dommages ne sont pas non plus identiques ou comparables sur toutes les communes demanderesses. Quant à la valeur des pluies, elle n'est pas généralisable à l'ensemble des communes inondées. Il est à cet égard intéressant de souligner la proposition Biwer en vue d'une prochaine réforme du système d'indemnisation : une définition de la catastrophe à l'échelle de la zone constatée comme affectée, soit à l'échelle infra-communale.

À ces problèmes s'ajoutent des éléments dont nous ne pouvons mesurer les effets sur la base de données. Nous pouvons mentionner par exemple les conséquences du changement de mentalités des élus face au régime d'indemnisation : un effet "nouveauté » au départ; une reconnaissance perçue ensuite comme une tentative de blocage du pouvoir d'urbanisation pour certains maires; puis une pression des particuliers aujourd'hui largement véhiculée à travers les médias. Plusieurs mesures prises depuis 1983 remettent aussi en cause l'homogénéité des données : le changement des franchises imposé dans le Code des assurances en 1995; la mise en place des Plans de prévention des risques en 1995 ; l'arrivée des Dossiers départementaux des risques majeurs, les Dossiers d'intérêt communal sur les risques majeurs et les Dossiers communaux synthétiques en 1997 afin d'améliorer la prise en compte des risques; ou encore la récente modification du Code des Assurances en $2000^{5}$. La base «CatNat» est directement affectée par tous ces changements qui sont connus mais non mesurables. Elle ne peut pas, en tout cas, servir de base de données statistique fiable sans la moindre modification.

\section{Affiner la méthode pour une meilleure évaluation des « crues rapides »}

\section{COMMENT PALLIER LES LACUNES DE LA BASE DE DONNÉES?}

Une des façons de pallier les limites de la base de données est de consulter les dossiers présentés devant la commission interministérielle qui statue sur la prise de décision. L'idée est de consulter les dossiers dans le détail pour ne garder que les « crues rapides liées aux orages » et en mesurer à la fois l'ampleur et la gravité. Les dossiers "Catnat » sont toutefois protégés et ne sont communicables au public qu'à l'expiration d'un délai de 30 ans, conformément aux conditions prévues par la loi sur les archives du 3 janvier 1979. Une convention « Recherche » a néanmoins été possible conformément aux règles de communication des archives publiques et avec l'accord de la DDSC (Direction de la Défense et Sécurité Civile) rattachée au ministère de l'Intérieur, les Archives nationales, la mission Patrimoine du ministère de la Culture et le CAC (Centre des archives contemporaines). L'analyse des fiches et des rapports techniques compilés dans les dossiers nous a permis d'obtenir les informations suivantes (fig. 5) :

5. La franchise est désormais modulée en fonction du nombre d'arrêtés pris pour le même risque sur les 5 années précédant la demande de reconnaissance; lorsque la commune a x arrêtés, la franchise est multipliée par $\mathrm{x}$ - 1 . 
- sur les demandes de reconnaissance figurent les estimations des dommages, la nature du sinistre, les dates des précédents arrêtés ainsi que les mesures de prévention prises sur la commune (travaux, aménagements, Plan de Prévention des Risques);

- les rapports circonstanciés des maires détaillent le sinistre. Les comptes rendus sont subjectifs mais la description de l'événement permet d'en supposer l'ampleur.

- le rapport météorologique (Météo France) donne les intensités de pluies relevées par les stations ainsi que les cumuls de pluie estimés par les échos-radars. Des durées de retour calculées à partir d'une station de référence sont également consultables. Ces données restent la propriété de Météo France et doivent être utilisés avec leur accord.

- le rapport de la direction régionale de l'environnement (DIREN) donne des valeurs de débit lorsque des stations ont pu mesurer les variations sur des cours d'eau équipés.

- celui de la direction départementale de l'équipement (DDE) apporte des estimations financières sur les dommages subis par les réseaux d'évacuation, les routes et autres.

- un «fonds de dossier» contient d'autres éléments suivant la gravité de l'épisode : les rapports de la gendarmerie et des services départementaux d'incendie et de secours (SDIS) où sont mentionnés le nombre, les lieux d’interventions et la nature des dégâts; les dépêches et coupures de presse des journaux locaux ou régionaux; les factures établies par les particuliers ; les photographies des dommages envoyées par les particuliers; et quelquefois, des cartes mentionnant les zones inondées.

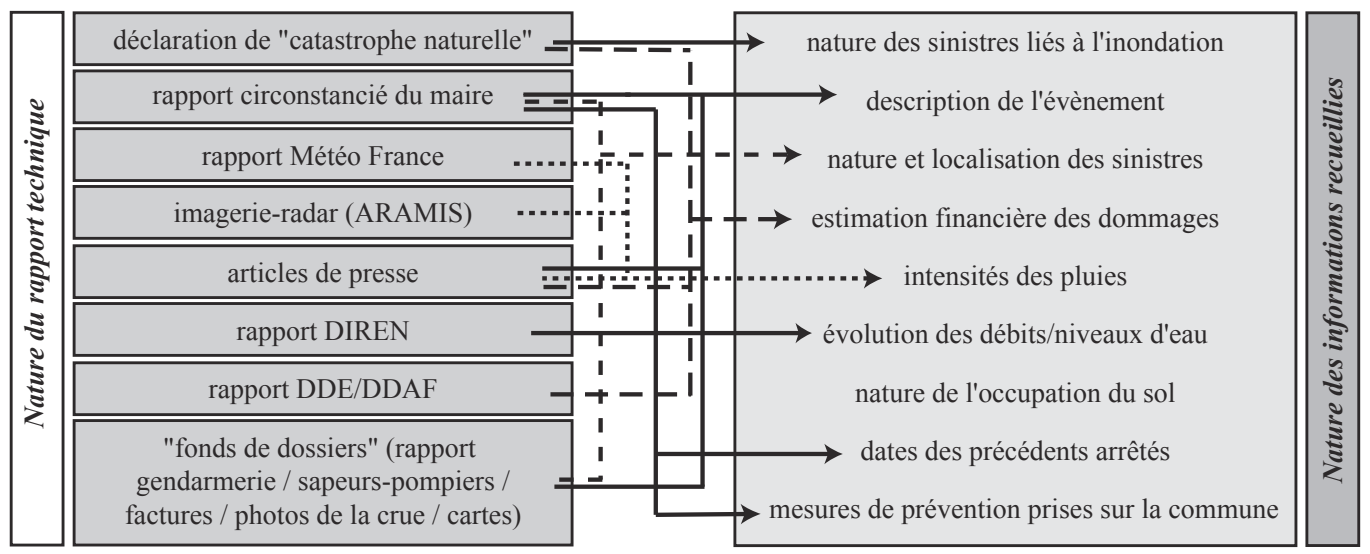

Figure 5 : Nature des rapports techniques et des informations issues des dossiers "CatNat » Information and technical reports from the French "CatNat" files

Les dossiers présentés devant la commission pour des épisodes reconnus entre mai et août sur la période 1983-2005 ont été analysés. Seules les informations concernant les «crues rapides » liées à de violents orages ont été conservées; elles permettent d'évaluer l'importance des sinistres et la gravité des dommages subis par les communes pour de tels événements.

\section{LES DOSSIERS « CATNAT » COMME SUPPORT D’ANALYSE POUR UN INVENTAIRE DÉTAILLÉ ET PRÉCIS DES INONDATIONS DE TYPE « CRUES RAPIDES »}

L'analyse détaillée des dossiers corrige les erreurs dues à l'architecture de la base de données et apporte un regard d'expert sur la prise de décision. Les événements de type «crues rapides » sont différenciés des autres inondations initialement comptabilisées et les communes touchées sont désormais celles qui ont réellement subi les assauts des ruissellements concentrés violents liés 
aux «crues rapides ». Même si le traitement des informations n'est pas exhaustif, cette seconde approche améliore considérablement l'analyse statistique initiale (tableau 2).

$31 \%$ des épisodes orageux reconnus ont réellement déclenché des «crues rapides ", soit 114 orages sur les 367 de départ. Attention : certains épisodes sont sans doute comptés en double car communs à plusieurs départements. Les écarts entre les données sont importants. Alors que $82 \%$ des épisodes reconnus pour la Seine-Maritime sont gardés, seuls $8 \%$ et $12 \%$ d'entre eux le sont respectivement pour le Nord et le Calvados. Ces résultats confirment la supposition de départ, à savoir que la majorité des orages déclenchent des stagnations d'eaux pluviales ou du ruissellement rural ou urbain dans ces deux régions, mais très peu de « crues rapides », à l'inverse des épisodes reconnus en Seine-Maritime.

L'opération est plus délicate à l'échelle des communes : 192 déclarations (sur les 3568 de départ) concernent uniquement des « crues rapides » (tableau 2). On garde ainsi seulement 7,4\% des communes initialement comptabilisées, avec des écarts qui vont de $2 \%$ pour le Nord et le Calvados à $13 \%$ pour le Pas-de-Calais et la Seine-Maritime. Cette dernière ressort comme la région de loin la plus sensible : elle regroupe 93 des 192 communes déclarées. Les chiffres doivent à nouveau être utilisés avec précaution car des communes comptées une seule fois ont en réalité été touchées par plusieurs épisodes. Le fait de n'avoir que si peu d'écart entre les départements est par contre assez surprenant. Deux raisons nous viennent à l'esprit. D'un côté, il est sûrement plus facile pour les communes des départements les moins touchés de se faire reconnaître en état de «catastrophe naturelle »; un souci d'équité en matière d'aménagement lisserait donc les disparités régionales. D’un autre côté, trop de demandes sont probablement accordées lors des épisodes les plus violents : soit en raison d'un manque de tri parmi toutes les demandes; soit à cause de la généralisation des données de pluies sur plusieurs communes.

\begin{tabular}{|c|c|c|c|c|c|c|c|c|c|}
\hline & 14 & 76 & 27 & 80 & 60 & 62 & 59 & 02 & $\begin{array}{r}\text { Moyenne } \\
\text { (total) }\end{array}$ \\
\hline $\begin{array}{l}\text { Nombre d'épisodes } \\
\text { recensés au départ (B) }\end{array}$ & 26 & 42 & 36 & 36 & 57 & 55 & 52 & 61 & $\begin{array}{r}45,6 \\
(367) \\
\end{array}$ \\
\hline $\begin{array}{l}\text { Épisodes du type } \\
\text { «crues rapides }(\mathrm{D})\end{array}$ & 3 & 36 & 6 & 9 & 16 & 20 & 4 & 25 & $\begin{array}{r}14,9 \\
(119) \\
\end{array}$ \\
\hline $\begin{array}{l}\text { Nombre d'épisodes } \\
\text { gardés }(\mathrm{D} / \mathrm{B})\end{array}$ & $12 \%$ & $82 \%$ & $17 \%$ & $24 \%$ & $28 \%$ & $36 \%$ & $8 \%$ & $41 \%$ & $31 \%$ \\
\hline $\begin{array}{l}\text { Déclarations entre mai } \\
\text { et août au départ }(\mathrm{A})\end{array}$ & 311 & 720 & 294 & 309 & 424 & 424 & 472 & 614 & $\begin{array}{r}446 \\
(3568)\end{array}$ \\
\hline $\begin{array}{l}\text { Déclarations pour les } \\
\text { «crues rapides }(\mathrm{C})\end{array}$ & 7 & 93 & 21 & 13 & 33 & 54 & 8 & 63 & $\begin{array}{r}36,5 \\
(192)\end{array}$ \\
\hline $\begin{array}{l}\text { Nombre Déclarations } \\
\text { gardées }(\mathrm{C} / \mathrm{A})\end{array}$ & $2 \%$ & $13 \%$ & $7 \%$ & $4 \%$ & $8 \%$ & $13 \%$ & $2 \%$ & $10 \%$ & $7,4 \%$ \\
\hline $\begin{array}{l}\text { Nombre de bassins } \\
\text { versants touchés }(\mathrm{E})\end{array}$ & 7 & 49 & 16 & 10 & 24 & 37 & 5 & 39 & $\begin{array}{r}23,4 \\
(187)\end{array}$ \\
\hline
\end{tabular}

Tableau 2 : Écarts obtenus entre les résultats après l'analyse détaillée des dossiers « CatNat » (seconde analyse) et ceux issus du traitement initial de la base statistique

Variation between the results obtained after the detailed analysis of the "CatNat" files (second analysis) and those resulting from the first treatment of the statistical data base

Les informations ont par ailleurs été recensées à l'échelle des bassins-versants (fig. 6). La carte montre que les phénomènes sont nombreux (187 bassins versants touchés) même s'ils restent peu fréquents à l'échelle de la zone d'étude (8,5 bassins touchés par an sur $50000 \mathrm{~km}^{2}$ ). Ces derniers se situent majoritairement dans le Pays de Caux, sur les plateaux de l'Artois, du Pernois, du Valois et du Vexin normand, dans le Ponthieu et le Tardenois. Des configurations spatiales originales semblent d'ailleurs ressortir car certains bassins se situent le long de vallées encaissées de cours d'eau importants (la Seine, la Marne) ou secondaires (la Risle, l’Authie, la Canche, le Troësne). 
Ces résultats confirment des hypothèses déjà suggérées à une échelle plus grande (Delahaye, 2002 ; Douvinet et al., 2006a). L'inventaire permet aussi de différencier les phénomènes de ruissellement localisé, qui sont des inondations par du ruissellement rural lié à une trop forte proximité cultures/habitations, des véritables «crues rapides » aux écoulements bien plus violents et dont les conséquences sont parfois dramatiques (Douvinet et al., 2006b).

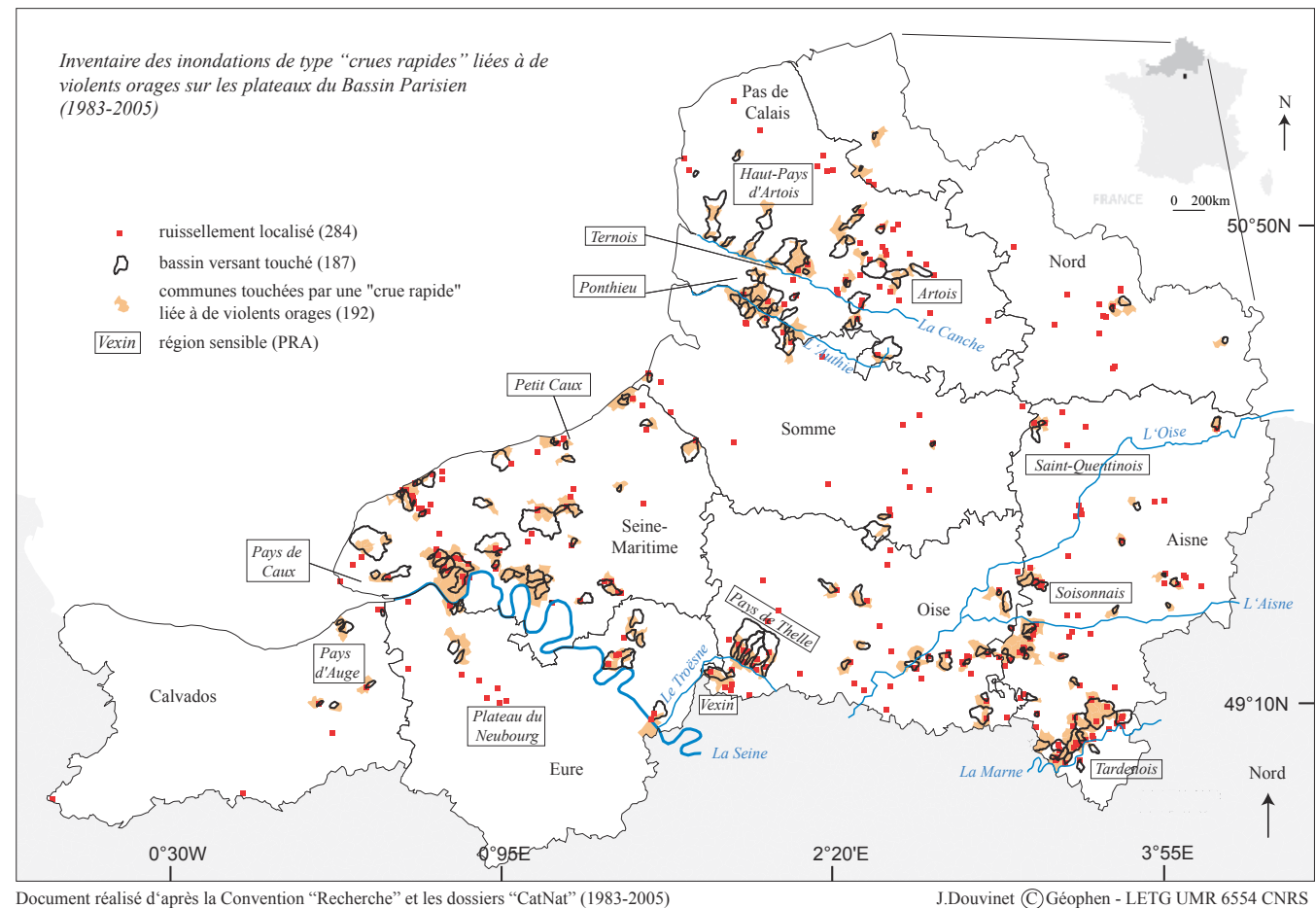

Figure 6 : Cartographie des bassins versants touchés par des épisodes de « crues rapides » liées à de violents orages à l'échelle des régions de plateaux du Bassin parisien (1983-2005)

Mapping of sensitive catchments and flooded places due to "flash floods" events linked to violent thunderstorms on the plateaux of the Paris basin over the period 1983-2005

Les régions touchées étaient déjà identifiées sur la première carte (fig. 3). Toutefois, la seconde carte est plus précise (fig. 6). Les informations sont recensées à l'échelle des entités hydrologiques fonctionnelles et les bassins versants situent les zones où l'aléa "crue rapide » a provoqué des dégâts sur les sociétés y étant implantées. A contrario, la première carte prenait uniquement en compte la vulnérabilité des populations sans distinguer les « crues rapides » des autres processus hydrologiques regroupés dans l'item « inondations et coulées de boue ».

\section{Conclusion}

Un inventaire complet, détaillé et non exhaustif des inondations par « crues rapides » liées à de violents orages est proposé pour les régions de plateaux du Bassin parisien à partir des données «CatNat». Cette démarche permet à la fois de dresser un état des lieux sur ces régions et de dépasser le cadre monographique des études de cas réalisées jusqu'à présent. La méthode d’inventaire utilisée se décompose en deux étapes indissociables. Les résultats de l'analyse statistique initiale de la base de données donnent des estimations sur la vulnérabilité de certains départe- 
ments; les données spatialisées ciblent les communes aux déclarations les plus nombreuses. Les limites inhérentes aux données et aux objectifs réglementaires de la procédure « CatNat » nous obligent toutefois à poursuivre l'analyse jusqu'à la consultation dans le détail des dossiers consultés pour la reconnaissance. Au final, ces dossiers se révèlent être de bons supports d'analyse et les informations tirées des rapports techniques contribuent à une évaluation pertinente des sinistres causées par des « crues rapides».

Ce travail d'inventaire est donc parti d'une base de données statistique pour arriver à une cartographie complète et détaillée des inondations à l'échelle des bassins versants. D'autres démarches existent, qu'elles soient basées sur des retours d'expérience (Garry, 1995; Gaume et al., 2000) ou, plus rarement, sur une approche historique (Ballais et Ballais, 1984; Laganier et al, 2000 ; Agasse, 2005). Ces méthodes d'inventaire peuvent aussi être complétées par les informations tirées des médias et autres sources journalistiques (Hay-Lepêtre, 2005). L'avantage apporté ici par les données «CatNat» est de travailler sur des événements dommageables et directement sur la sinistralité des inondations affectant les populations (Pigeon, 2002). De nouvelles pistes apparaissent suite à ce premier travail. En effet, des « crues rapides » sont-elles apparues sur des vallons où les sociétés ne sont pas implantées? À l'inverse, la vulnérabilité des populations au sein des bassins est-elle le seul facteur aggravant? Dès lors, comment interpréter la carte obtenue à partir de l'analyse des dossiers « CatNat »?

\section{Bibliographie}

Agasse (E.), 2005. - Les crues de rivières en Basse-Normandie. Expression d'un système hydrogéographique complexe en milieu tempéré océanique, Thèse de Géographie, université de Caen Basse-Normandie, 553 p.

Angeliaume (A.), WichereK (S.), 1997. - «Contribution d'un bassin-versant élémentaire cultivé à la formation des crues ", Géomorphologie : relief, processus, environnement, n² 2, p. 155-167.

Auzet (A.-V.), Boiffin (J.), LudWig (B.), 1995. - «Érosion hydrique dans les bassins versants agricoles des régions limoneuses du nord-ouest de la France », Annales de Géographie, nº 281/282, p. 187-190.

Ballais (H.), Ballais (J.-L.), 1984. - « Éléments pour une histoire des inondations dans le Calvados au $\mathrm{XVIII}^{\mathrm{e}}$ et XIX ${ }^{\mathrm{e}}$ siècle d'après les archives départementales », Travaux du centre de recherche en géographie physique de l'environnement, Vol. 5, Université de Caen Basse-Normandie, p. 19-44.

Boardman (J.), Evans (R.), Ford (J.), 2003. - « Muddy floods on the South Downs, southern England: problem and responses ", Environmental Sciences \& Policy, vol. 6, p. 69-83.

Cerdan (O.), Le Bissonnais (Y.), Couturier (A.), Bourennane (H.), Souchère (V.), 2002. - « Rill erosion on cultivated hillslopes during two extreme rainfall events in Normandy, France », Soil E Tillage Research, vol. 67, p. 99-108.

Dauphine (A.), 2001. - Risques et catastrophes. Observer, spatialiser, comprendre, gérer, Paris, Armand Colin, coll. «U $», 288$ p.

Delahaye (D.), 1999. - « Originalité des risques hydrologiques; La catastrophe de Saint-Martin-de-Boscherville en juin 1997 », Études Normandes, vol. 1-2, p. 157-170.

—, 2002. - Apport de l'analyse spatiale en Géomorphologie. Modélisation et approche multiscalaire des risques. Mémoire d'habilitation à diriger des recherches, Université de Rouen, $471 \mathrm{p}$.

Douvinet (J.), Delahaye (D.), LAnglois (P.), 2006a. - « Les apports de l'IAD à la caractérisation morphométriques des bassins versants sensibles aux crues rapides hyper-concentrées (Bassin parisien, Nord de la France) », Proceedings of the International Conference on "Interactions Nature-Sociétés. Analyse et modèle », La Baule, CD-ROM, 6 p.

—, 2006b. - «Application of cellular automata modelling to analyse the dynamics of hyper-concentrated stream flows on loamy plateaux (Paris Basin, North-western France) », Proceedings of the $7^{\text {th }}$ International Conference on HydroInformatics, "Innovate and share », Nice (France), Volume 2, p. 1088-1094. 
GARRY (G.), 1995. - «Auch, Nîmes, Vaison-la-Romaine : retours d'expériences », Bulletin de l'Association des Géographes Français, BAGF, Paris, p. 131-145.

Gaume (E.), Livet (M.), Pons (M.), 2000 - Analyse du comportement hydrologique des parties amont des bassins versants lors des crues des 12 et 13 novembre 1999 dans l'Aude, les Pyrénées Orientales et le Tarn. Rapport de synthèse pour le MATE (ministère de l'Aménagement du Territoire et de l'Environnement), 20 p.

Hauchard (E.), Delahaye (D.), Frankhauser (P.), 1999. - «Analyse morphologique des talwegs et comportement scalant. Applications des dynamiques non entières à la géographie physique », L'espace géographique, vol. 3, p. 215-224.

Hay-Lepetre (C.), 2005. - Dynamiques spatiale et temporelle de l'aléa inondation: De l'analyse des phénomènes à l'évaluation des pratiques d'aménagement: L'exemple de la Seine-Maritime. Thèse de Doctorat, Université de Rouen, 530 p.

Laganier (R.), Piquet (P.), Salvador (P.-G.), Scarwell (H.-J.), 2000. - « Inondations, territoire et aménagement : l'évolution de la prise en compte du risque inondation dans la vallée de la Canche (Pas-de-Calais, France) », Géocarrefour, Revue de Géographie de Lyon, p. 375-382.

Le Bissonnais (Y.), Thorette (J.), Bardet (C.), Daroussin (J.), 2002. - L'érosion hydrique des sols en France, Rapport INRA-IFEN, 109 p.

LUDWIG (B.) 1992. - L'érosion par ruissellement concentré des terres cultivées du Nord du Bassin parisien. Analyse de la variabilité des symptômes d'érosion à l'échelle du bassin-versant élémentaire, Thèse de Doctorat, Université Louis-Pasteur, Strasbourg, 155 p.

MEYER (E.), 2001. - Évolution des paysages ruraux et phénomènes d'inondation boueuse en terres de grande culture. Le cas du ru de Senneville (Yvelines), Thèse de Géographie, Paris IV, Sorbonne, 413 p. + annexes.

Ministère de L’Aménagement du Territoire et de L'Environnement, 2001. - Inondations et coulées de boue en Seine-Maritime, Rapport de l'Inspection Générale de l'Environnement (IGE), 70 p. + annexes.

Ministère de l’Écologie et du Développement Durable, 2005. - Mission d'enquête sur le régime d'indemnisation des victimes des catastrophes naturelles, Rapport de l'Inspection Générale des Finances (IGF), du Conseil Général des Ponts et Chaussées (CGPC), et de l'Inspection Générale de l'Environnement (IGE), 95 p. + annexes.

Météo France, ministère de L'Écologie et du Développement Durable, 2004. - Pluies extrêmes sur le Nord de la France, CD-ROM.

Pigeon (P.), 2002. - «Réflexions sur les notions et les méthodes en géographie des risques dits naturels », Annales de Géographie, vol. 111, n 627-628, p. 452-470.

Cet article a été reçu le 19 septembre 2006 et définitivement accepté le 27 novembre 2006.

\section{REMERCIEMENTS}

Ce travail a été possible grâce au financement par le MRNT et le CNRS du programme ACI "Systèmes Complexes en SHS » intitulé "Analyse de la structure et de la dynamique de systèmes spatiaux complexes: les bassins versants sensibles aux crues rapides " (2004-2007). L'auteur tient à remercier Marie-Paule Couteron, Karine David, Anne-Laure Pierret, Jean Claude Hermet et Hervé Adeux (Direction de la défense et sécurité civile du ministère de l'Intérieur), François Giustiniani (exchargé de la Mission Patrimoine au sein du ministère de la Culture), Arnaud de Castelbajac (Mission des Archives Nationales), Pauline Moirez (Centre des Archives Contemporaines) et Fanny Cam sans qui la consultation des dossiers "CatNat " n'aurait jamais été possible. L'auteur remercie également Daniel Delahaye, Laurent Lespez et les relecteurs de la revue pour les corrections et les modifications apportées à l'article. 
